\title{
L6E9 Myoblasts Are Deficient of Myostatin and Additional TGF- $\beta$ Members Are Candidates to Developmentally Control Their Fiber Formation
}

\author{
Stefania Rossi, ${ }^{1}$ Elena Stoppani, ${ }^{1}$ Massimiliano Gobbo, ${ }^{2}$ Anna Caroli, ${ }^{3}$ \\ and Alessandro Fanzani ${ }^{1}$ \\ ${ }^{1}$ Unit of Biochemistry, Department of Biomedical Sciences and Biotechnologies, University of Brescia, \\ viale Europa 11, 25123 Brescia, Italy \\ ${ }^{2}$ Unit of Physiology, Department of Biomedical Sciences and Biotechnologies, University of Brescia, \\ viale Europa 11, 25123 Brescia, Italy \\ ${ }^{3}$ Unit of Biology and Genetics, Department of Biomedical Sciences and Biotechnologies, University of Brescia, \\ viale Europa 11, 25123 Brescia, Italy
}

Correspondence should be addressed to Alessandro Fanzani, fanzani@med.unibs.it

Received 6 October 2009; Revised 11 December 2009; Accepted 5 February 2010

Academic Editor: Sanford I. Bernstein

Copyright (C) 2010 Stefania Rossi et al. This is an open access article distributed under the Creative Commons Attribution License, which permits unrestricted use, distribution, and reproduction in any medium, provided the original work is properly cited.

This work provides evidence that the robust myoblast differentiation observed in L6E9 cells is causally linked to deficiency of myostatin, which, conversely, has been found to be expressed in $\mathrm{C} 2 \mathrm{C} 12$ cells. However, despite the absence of endogenous myostatin, L6E9 myoblasts expressed functional Activin receptors type II (ActRIIs) and follistatin as well as the highly related TGF- $\beta$ members Activins and GDF11, suggesting that in this cell line the regulation of fiber size might be under the control of multiple regulators regardless of myostatin. In line with this hypothesis, delivery of a dominant-negative ActRIIb form or the increase of follistatin, as obtained via Trichostatin treatment or stable transfection of a short human follistatin form, enhanced the L6E9 cell differentiation and further increased the size of myotubes, suggesting that L6E9 myoblasts provide a spontaneous myostatin knock-out in vitro model to study TGF- $\beta$ ligands involved in developmental regulation of fiber size.

\section{Introduction}

Over the last years, the TGF- $\beta$ member myostatin has gained particular relevance because of its ability to exert a profound effect on muscle metabolism, by regulating the myofiber size in response to physiological or pathological conditions [1-5]. Of note, myostatin loss-of-function due to naturally occurring mutations into its gene triggers muscle mass increase in cattle [6], dogs [7], and humans as well [8], whereas targeted disruption of myostatin gene produces a huge muscle mass in mice [1]. On the contrary, systematic administration of myostatin induces muscle cachexia [9], and several conditions which cause muscle atrophy enable increase of myostatin expression [10-12]. Therefore, reduced or excessive myostatin signaling affects the muscle metabolism by inducing muscle hypertrophy and atrophy, respectively. Normally, myostatin signals in myoblasts through a canonical TGF- $\beta$ signaling pathway, that occurs after binding with Activin receptors (ActRIIs) [3] and the subsequent activation of a Smad ternary complex $[13,14]$, which in turn drives to a transcriptional program potentially involved in muscle remodeling. In line with this evidence, the block of myostatin pathway in mice by delivering a dominant-negative ActRIIb form triggers an increase of muscle mass [15]. On the other side, follistatin has been described as a powerful inducer of muscle mass, due to its ability to bind and neutralize the myostatin activity [15-18]. However, follistatin transgenic mice display bigger muscles than myostatin null mice [15], and breeding transgenic follistatin mice with myostatin null mice triggers quadrupling of muscle mass [19], suggesting that follistatin can promote muscle growth also independently of its action 
on myostatin. To date, most of the in vitro myoblast studies relied on the use of mouse $\mathrm{C} 2 \mathrm{C} 12$ and rat L6E9 cells, two immortalized lines whose myogenesis process recapitulates the phases of embryonic muscle differentiation, when myoblasts undergo alignment, fusion, and growth in the attempt to form a contractile myofiber. In this work, by comparing the differentiation between $\mathrm{C} 2 \mathrm{C} 12$ and L6E9 myoblasts, we hypothesize that the more robust formation of myotubes in L6E9 is causally linked to deficiency of myostatin, which is expressed in C2C12 myoblasts. Additionally, since we recognized that ActRIIs and follistatin are expressed in L6E9 cells, we verified whether the delivery of a dominantnegative ActRIIb form or the overexpression of follistatin might influence the differentiation and the development of L6E9 myotubes irrespective of myostatin. Finally, RT-PCR analysis was carried out to detect whether L6E9 cells express Activins $[20,21]$ and GDF11 $[20,22]$, which are TGF- $\beta$ members supposed to play redundant roles with myostatin to regulate the muscle mass.

\section{Materials and Methods}

2.1. Materials. All reagents were from Sigma-Aldrich, if not otherwise indicated.

2.2. Cell Cultures, Cell Staining, and Myotube Quantification. C2C12 and L6E9 myoblasts were maintained in humidified incubator at $37^{\circ} \mathrm{C}$ and $5 \% \mathrm{CO}_{2}$ in DMEM high glucose supplemented with $10 \%$ or $20 \%$ FBS, respectively, and $100 \mu \mathrm{g} / \mathrm{mL}$ penicillin-streptomycin. To induce differentiation, preconfluent $\mathrm{C} 2 \mathrm{C} 12$ and L6E9 myoblasts were cultured in a medium consisting of DMEM supplemented with $2 \%$ HS or $1 \%$ FBS, respectively. To visualize myotubular structures, cells were fixed in methanol and stained with Giemsa reactive. To quantify the myotube size, an Image ProPlus software (version 6.2) was used; the average size of myotubes was the mean of 100 measurements, as obtained after measuring 10 myotubes in 10 different fields.

2.3. Drug Treatments. Differentiated L6E9 myotubes were treated with $100-500 \mathrm{ng} / \mathrm{mL}$ human-purified recombinant myostatin (BioVision). Trichostatin A ( $50 \mathrm{nM})$ was administered to preconfluent cells up to $16-18$ hours before addition of the differentiation medium.

2.4. Animals. Experiments were performed according to the European Community Council Directive, November 1986 (86/609/EEC). Wistar rats and C57BL/6 mice were anesthetized with chloral hydrate $(400 \mathrm{mg} / \mathrm{kg})$ and then sacrificed. Gastrocnemius muscles were collected and immediately frozen by liquid nitrogen before proceeding with RNA isolation.

2.5. RNA Isolation and Semiquantitative RT-PCR Analysis. Total RNA was isolated by Trireagent method, digested with DNase (DNA-free, Ambion), and reverse transcribed $(2 \mu \mathrm{g})$ in the presence of 400 units of MMLV-RT (Promega). The following primers (forward and reverse, $250 \mathrm{nM}$ each) were designed to amplify both the mouse and rat transcripts.

(i) Activin A (accession number NM_008380), 5' -TTCTGTTGGCAAGTTGCTGGATTA- $3^{\prime}$ and $5^{\prime}$-AAAGGTGATGATCTCCGAGGTCTG-3',

(ii) Activin B (accession number NM_008381), 5' TCGTCTCTAACGAAGGCAACC- $3^{\prime}$ and $5^{\prime}$-ATGAGCCGAAAGTCGATGAAGAAC-3',

(iii) Activin receptor type IIa (accession number NM_ 007396), 5' -GCCCACTTCAAATCCTGTTACACC$3^{\prime}$ and $5^{\prime}$-ATCCACACTGGTGCCTCTTTTCTC- ${ }^{\prime}$,

(iv) Activin receptor type IIb (accession number NM 007397), 5' -CTCGGGAGTGCATCTACTACAACG$3^{\prime}$ and $5^{\prime}$-TTCCGATGACGATACATCCAGAAG-3',

(v) Follistatin (accession number NM_008046), 5' -CTCTTCAAGTGGATGATTTTC- $3^{\prime}$ and $5^{\prime}$-ACAGTAGGCATTATTGGTCTG-3',

(vi) Glyceraldehyde-3-phosphate dehydrogenase (gapdh, accession number NM_008084), 5'-GGTGCTGAGTATGTCGTGGAGTC- $3^{\prime}$ and $5^{\prime}$-GGACTGTGGTCATGAGCCCTTCC-3',

(vii) Growth differentiation factor 11 (GDF11, accession number NM_010272), 5'-AGAGGACGAGTACCACGCTACCAC-3' and 5'-CACTTGCTTGAAGTCGATGCTCTG-3',

(viii) Myostatin (accession number NM_010834), 5' -AACCCATGAAAGACGGTACAAGGT- $3^{\prime}$ and 5'-ATCTTTTGGGTGCGATAATCCAGT-3',

(ix) p21 (accession number NM_007669), 5'-GAGAACGGTGGAACTTTGACTTCG-3' and 5'-GCGCTTGGAGTGATAGAAATCTG-3' .

Levels of transcript amplification were quantified by using Image ProPlus software version 6.2, after normalization with the gapdh transcript.

2.6. Myoblast Transfection. Transfections were carried out by using Lipofectamine 2000 reagent (Invitrogen), according to manufacturer's instructions. L6E9 cells were stably transfected with a MDAF2 vector containing the myosin light chain promoter and $1 / 3$ enhancer and simian virus 40 processing sites, harboring a truncated dominant negative form of murine Activin receptor type IIb that lacks the kinase domain (dnActRIIb, aminoacids 1-174) [15], together with a pBabe plasmid harboring a puromycin gene resistance. L6E9 cells were also transfected with a pBabe/follistatin vector, and transfectants were obtained after antibiotic selection $(2 \mu \mathrm{g} / \mathrm{mL})$.

2.7. Immunoblotting. Protein concentration was obtained by bicinchoninic acid assay (Pierce). Total homogenates were obtained by harvesting the myoblasts in cold RIPA lysis buffer ( $1 \%$ Nonidet P40, $0.5 \%$ sodium deoxycholate, $0.1 \%$ SDS in $50 \mathrm{mM} \mathrm{NaCl}, 20 \mathrm{mM}$ Tris- $\mathrm{HCl} \mathrm{pH}$ 7.6), containing a mix of protease inhibitors (Roche). Samples were separated 
by SDS-PAGE under reducing conditions and transferred to nitrocellulose membranes. The following primary mouse monoclonal antibodies (1:1000 diluted) were used: antiMyogenin (clone F5D, Santa Cruz Biotechnology), antiMyHC (Hybridoma Bank, University of Iowa), and antiCav-3 (clone 26, BD Transduction Laboratories). A diluted $1: 10000$ antibody against tubulin (Sigma-Aldrich) was used to normalize protein level content. Blots were then incubated with secondary antibodies conjugated with horseradish peroxidase (Chemicon) and Western Blots revealed with enhanced chemiluminescence (Chemicon).

2.8. Statistics. All data are expressed as mean \pm SEM. Statistical significance was determined using $t$-Student analysis. A $P$ value $<.05$ was considered significant.

\section{Results and Discussion}

3.1. L6E9 Myoblasts Provide a Spontaneous Myostatin KnockOut "In Vitro" Model. In this study we first compared the extent of differentiation between the mouse $\mathrm{C} 2 \mathrm{C} 12$ and rat L6E9 myoblasts, two cell lines that are commonly used for myogenesis studies. After 2 and 4 days of low-serum treatment, the myotubes appeared larger in L6E9 compared to $\mathrm{C} 2 \mathrm{C} 12$ cells, as morphologically visualized by phase contrast images (Figure 1(a)). A graphical representation shows that, after 4 days, the average size of L6E9 myotubes reached about twofold of increase compared to C2C12 myotubes (Figure 1(b)). Throughout the differentiation, the protein levels of the muscle-specific markers myogenin, Caveolin 3 (Cav-3), and Myosin heavy chain (MyHC) increased earlier in L6E9 compared to $\mathrm{C} 2 \mathrm{C} 12$ cells, as detected by immunoblotting (Figure $1(\mathrm{c})$ ), suggesting that the fusion process proceeds more rapidly in L6E9 cells. Subsequently, we investigated whether the different behavior of $\mathrm{C} 2 \mathrm{C} 12$ and L6E9 myoblasts might reflect different expression levels of myostatin and follistatin, two secreted TGF- $\beta$ family members that exert profound and opposite effects on muscle mass, being a negative and positive regulator of muscle size, respectively [15]. By semiquantitative RT-PCR analysis, we detected that $\mathrm{C} 2 \mathrm{C} 12$ cells express increasing levels of both myostatin and follistatin during differentiation, whereas L6E9 myoblasts lack myostatin despite the evident increase of follistatin (Figure 1(d)). To rule out the possibility that the primers designed for amplifying myostatin were not specific for the rat isoform, we performed an RT-PCR by using samples obtained from mouse and rat gastrocnemius muscles. As shown in Figure 1(e), the primers specifically produced the $377 \mathrm{bp}$ long fragment of myostatin in both mouse and rat tissues, confirming therefore that L6E9 cells lack endogenous expression of myostatin. Additionally, we verified that both the cell lines constitutively express the transcripts for Activin receptors (ActRIIs), type IIa and IIb (ActRIIa and b) (Figure $1(\mathrm{~d})$ ), which are the TGF- $\beta$ receptors that bind myostatin with low and high affinity, respectively [15]. To prove whether ActRIIs receptors are functional in L6E9 cells, we next administered 100 and $500 \mathrm{ng} / \mathrm{mL}$ recombinant myostatin ( $\mathrm{r}-\mathrm{mstn}$ ) in the medium of differentiated L6E9 cells. Within 48 hours of treatment, r-mstn promoted a morphological impairment of myotube size compared to untreated cells, as shown by phase contrast images (Figure 2(a)). The graph in Figure 2(b) shows that r-mstn significantly decreased the size of L6E9 myotubes and triggered the downregulation of $\mathrm{MyHC}$ expression with a dose-dependent effect compared to untreated cells, as detected by immunoblotting (Figure 2(c)). It has been established that myostatin promotes the activation of both Smad and Foxo pathways by triggering its own transcription [23]. Therefore, we next controlled whether exogenous rmstn administration might restore the endogenous levels of myostatin in L6E9 cells. As suggested by RT-PCR analysis, endogenous myostatin transcript was undetectable in L6E9 myotubes even after treatment with $500 \mathrm{ng} / \mathrm{mL}$ r-mstn up to 48 hours (Figure 2(d)), whereas the transcript level of p21, a downstream target of myostatin pathway [24], was increased; under these conditions, follistatin expression was downregulated and the transcript levels for ActRIIa and IIb were unaffected (Figure 2(d)).

Collectively, these data show that the robust differentiation observed in L6E9 is characterized by deficiency of myostatin. Although the behavior of L6E9 cells cannot be exclusively ascribed to this hallmark sign, it is conceivable that these myoblasts take advantage of lack of myostatin and simultaneous presence of follistatin throughout the differentiation process. Additionally, L6E9 cells possess functional ActRIIs, given that exogenous administration of recombinant myostatin promoted myotube impairment. Since multiple TGF- $\beta$ ligands have been suggested to converge on these receptors [19], probably mimicking the action of myostatin, it is possible that the size of L6E9 myotubes might be under the control of additional regulators; in line with this hypothesis, L6E9 myoblasts express follistatin, which is known to antagonize multiple negative regulators of muscle mass [22].

3.2. Delivery of a Dominant-Negative ActRIIb Form or the Increase of Follistatin Levels Enhances the Size of L6E9 Myotubes Independently on Myostatin. It has been established that different TGF- $\beta$ proteins act to regulate the size of skeletal muscle mass in vivo. This evidence was first provided by the fact that follistatin transgenic mice display a muscle mass that is greatly increased compared to myostatin knock-out mice, suggesting that follistatin has the unrivalled ability to bind and neutralize the activity of additional myostatin-like regulators [19]. Therefore, we wanted to verify whether the size of L6E9 myotubes might be incremented irrespective of myostatin expression. To this purpose, L6E9 cells were stably transfected with a dominantnegative truncated ActRIIb form (dnActRIIb), which has been described to induce doubling of muscle mass in mice via abolition of myostatin signaling [15]. Additionally, in order to increase follistatin, we exposed L6E9 cells to treatment with the histone deacetylases inhibitor Trichostatin (TSA) [18] or delivered into L6E9 myoblasts a vector encoding a short human follistatin form. As shown by RT-PCR analysis carried out over a time-course differentiation of three days (Figure 3(a)), the amount of ActRIIb was remarkably increased in dnActRIIb transfectants compared to control, 


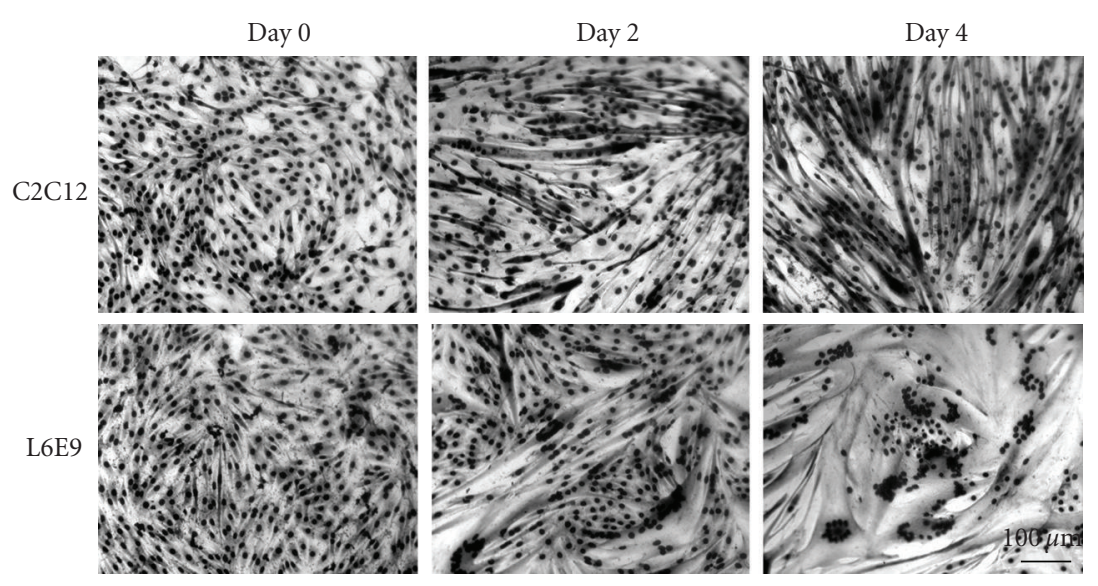

(a)

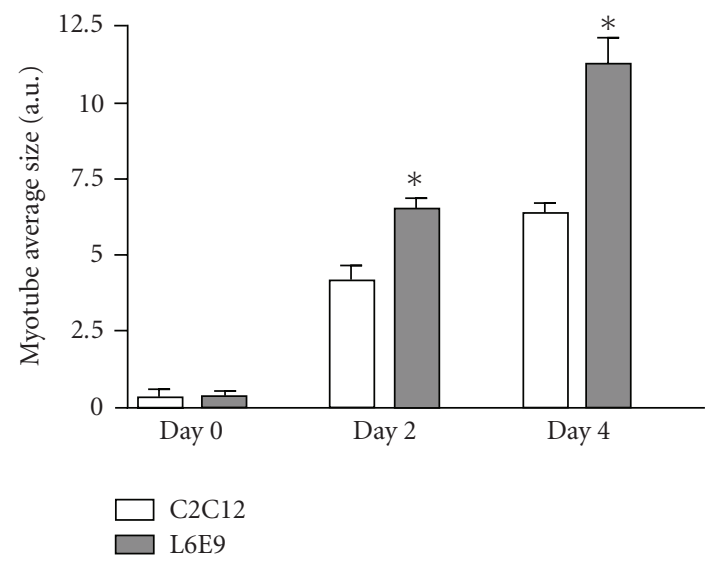

(b)

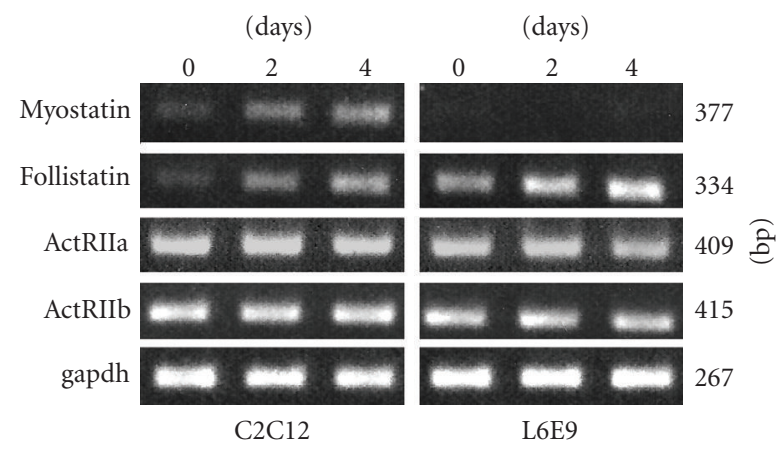

(d)

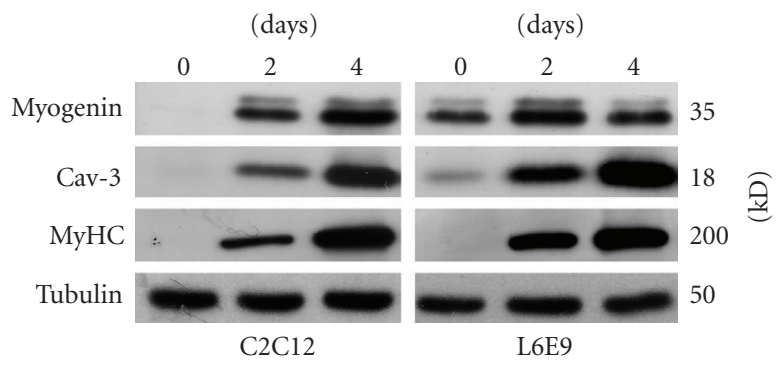

(c)

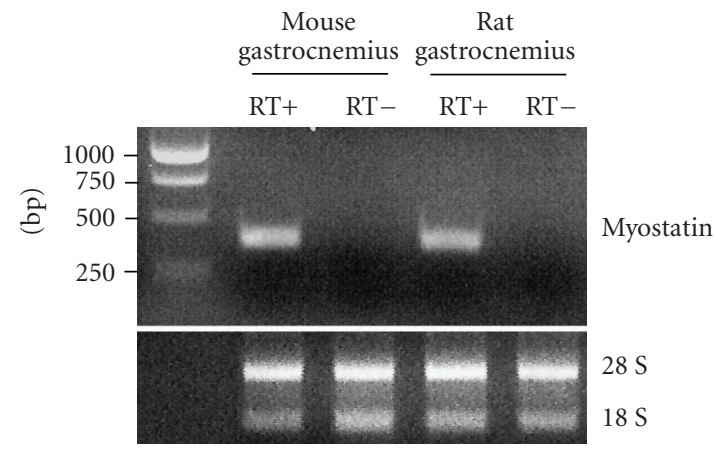

(e)

Figure 1: (a) Morphological visualization of C2C12 and L6E9 myoblasts undergoing differentiation. After Giemsa staining, phase contrast pictures were taken under the same magnification. Bar $=100 \mu \mathrm{m}$. (b) The graph represents the quantification of myotube average size in differentiating $\mathrm{C} 2 \mathrm{C} 12$ and L6E9 cells. ${ }^{*} P<.05$. (c) Immunoblotting was performed to compare the time-course expression of myogenin, Cav-3 and MyHC between C2C12 and L6E9 cells. Tubulin was used as loading control. (d) Semiquantitative RT-PCR analysis was performed to detect the transcript levels of myostatin, follistatin, ActRIIa, and ActRIIb in C2C12 and L6E9 cells cultured in differentiating medium. Gapdh amplification was performed as loading control. (e) RT-PCR analysis was carried out to amplify a 377 bp long fragment of myostatin in both mouse and rat gastrocnemius muscles. The amplification was performed by using the total RNA processed in presence or absence of reverse transcriptase (RT+ or RT-, resp.). Total RNAs are shown as loading control. 

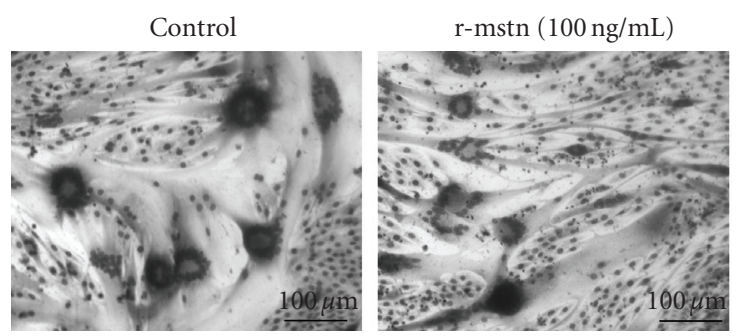

r-mstn $(500 \mathrm{ng} / \mathrm{mL})$

(a)

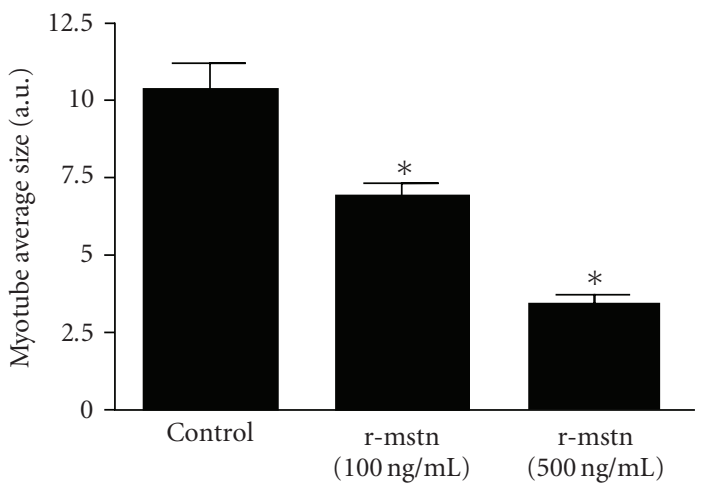

(b)

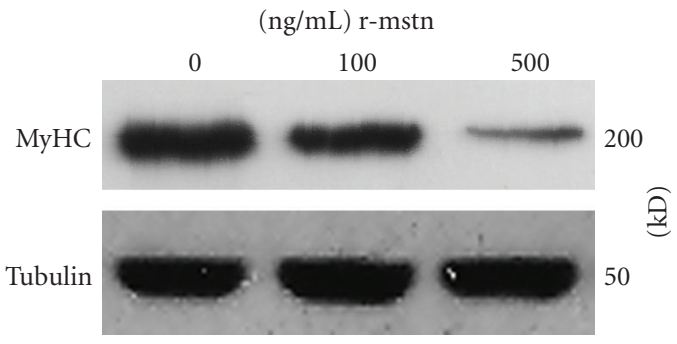

(c)

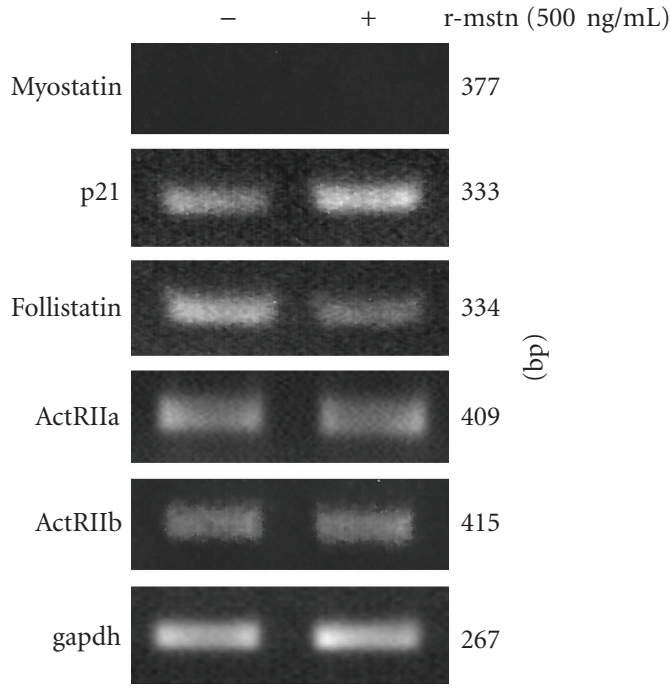

(d)

Figure 2: (a) Morphological visualization of L6E9 myotubes, untreated (control, day 5) or treated at day 3 with $100 \mathrm{or} 500 \mathrm{ng} / \mathrm{mL}$ recombinant myostatin (r-mstn) up to 48 hours, as depicted by phase contrast pictures after Giemsa staining. Bar $=100 \mu \mathrm{m}$. (b) The graph represents the quantification of average size in L6E9 myotubes, treated with 100 or $500 \mathrm{ng} / \mathrm{mL} \mathrm{r}$-mstn up to 48 hours as compared to control. ${ }^{*} P<.05$ versus control. (c) Immunoblotting shows that MyHC levels are downregulated in L6E9 myotubes exposed to r-mstn (100 or $500 \mathrm{ng} / \mathrm{mL}$ ) up to 48 hours as compared to untreated cells. Tubulin was used as loading control. (d) Semiquantitative RT-PCR analysis was performed to detect the expression levels of myostatin, p21, follistatin, ActRIIa, and ActRIIb in L6E9 myotubes (day 3) treated with r-mstn $(500 \mathrm{ng} / \mathrm{mL})$ up to 48 hours compared to untreated. Gapdh amplification was performed as loading control.

because of the combined amplification of both the fulllength endogenous and the truncated ectopic form. Under these conditions, dnActRIIb cells also displayed increased endogenous follistatin levels compared to control cells.
After TSA treatment or delivery of follistatin transgene, the levels of follistatin were strongly increased over the entire kinetic of differentiation compared to control cells, whereas the levels of ActRIIb remained unchanged (Figure 3(a)). 


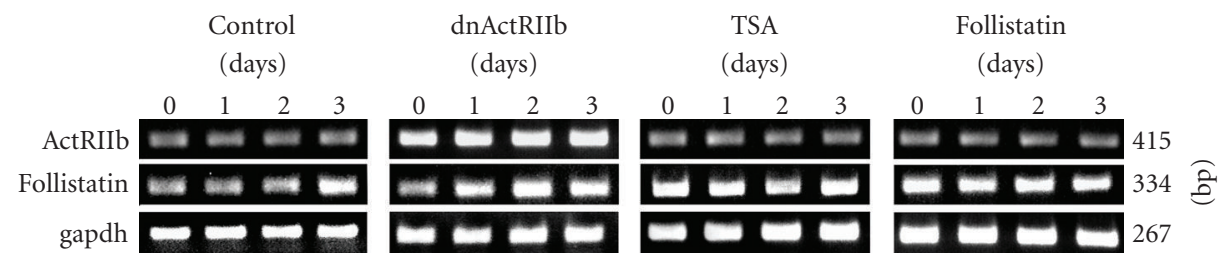

(a)

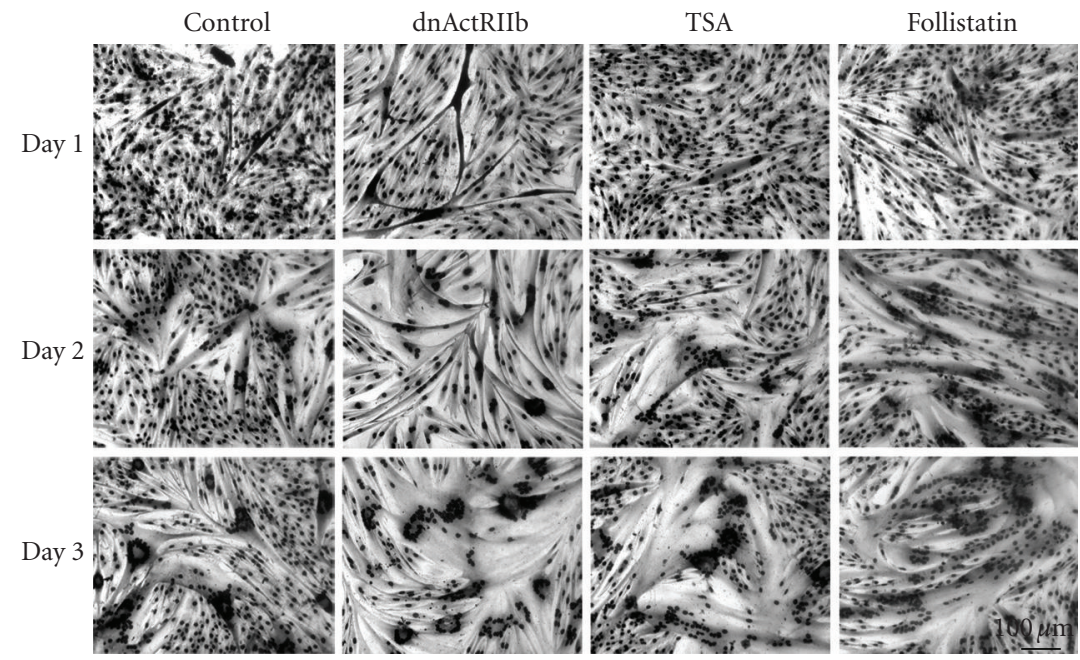

(b)
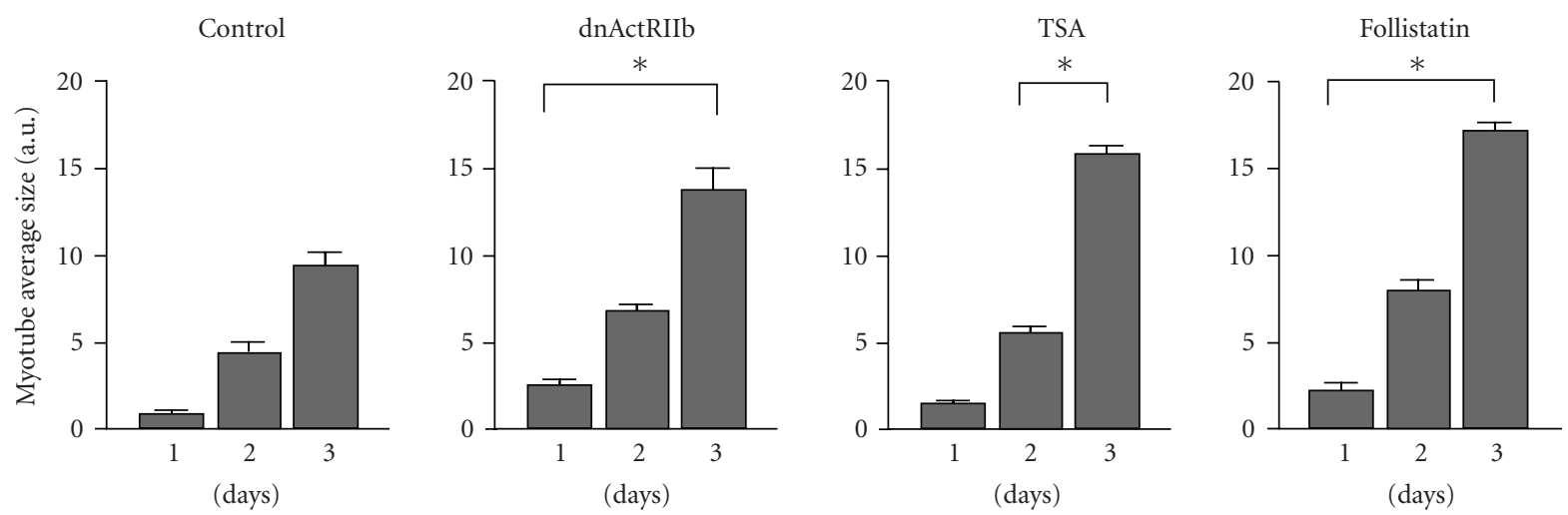

(c)
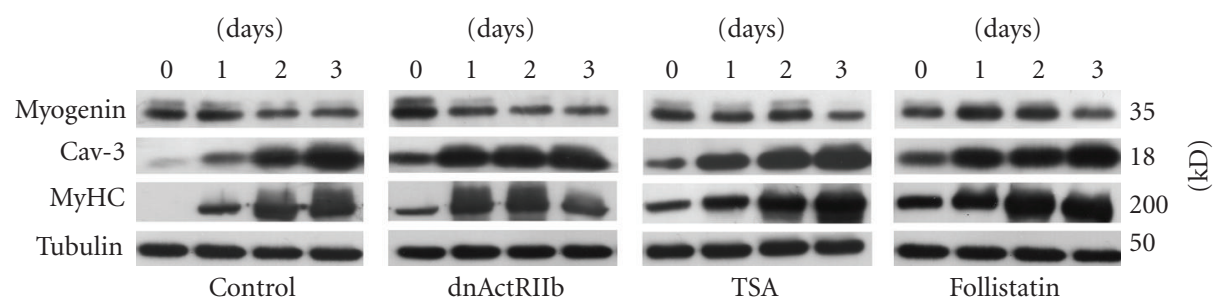

(d)

FIgure 3: (a) Semiquantitative RT-PCR analysis was carried out to detect the levels of ActRIIb and follistatin levels in untransfected L6E9 (control) and in cells stably transfected with the dnActRIIb form, treated with TSA, or alternatively transfected with a short human follistatin form. Gapdh amplification was performeds as loading control. (b) Phase contrast pictures show the morphology of L6E9 cells after delivery of a dnActRIIb form, exposure to histone deacetylases inhibitor TSA, or stable follistatin overexpression, as compared to control cells. After Giemsa staining, pictures were taken under the same magnification over a time-course of 3 days. Bar $=100 \mu \mathrm{m}$. (c) The graphs report the quantification of the myotube size in L6E9 cells transfected with dnActRIIb, treated with TSA or overexpressing follistatin as compared to control over a 3-day time-course differentiation. ${ }^{*} P<.05$ versus control. (d) Immunoblotting was performed to detect the protein levels of myogenin, Cav-3, and MyHC in L6E9 expressing the dnActRIIb form, exposed to TSA or alternatively transfected with follistatin compared to control cells. Tubulin was used as loading control. 


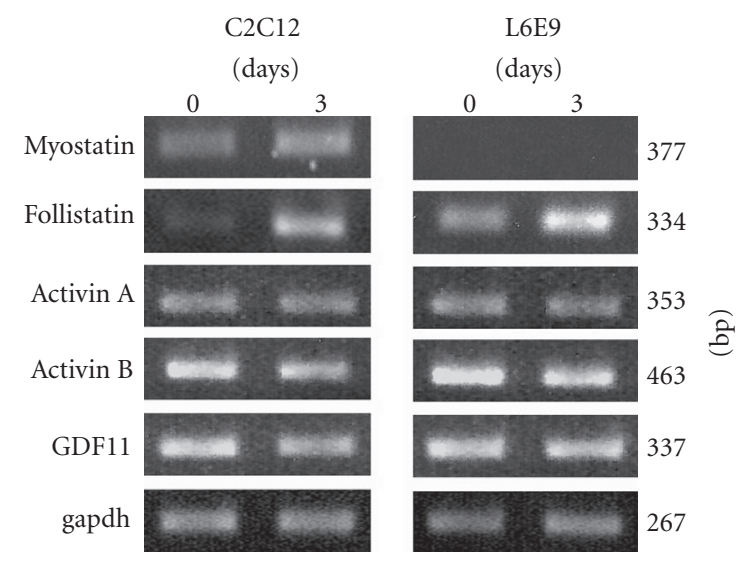

(a)
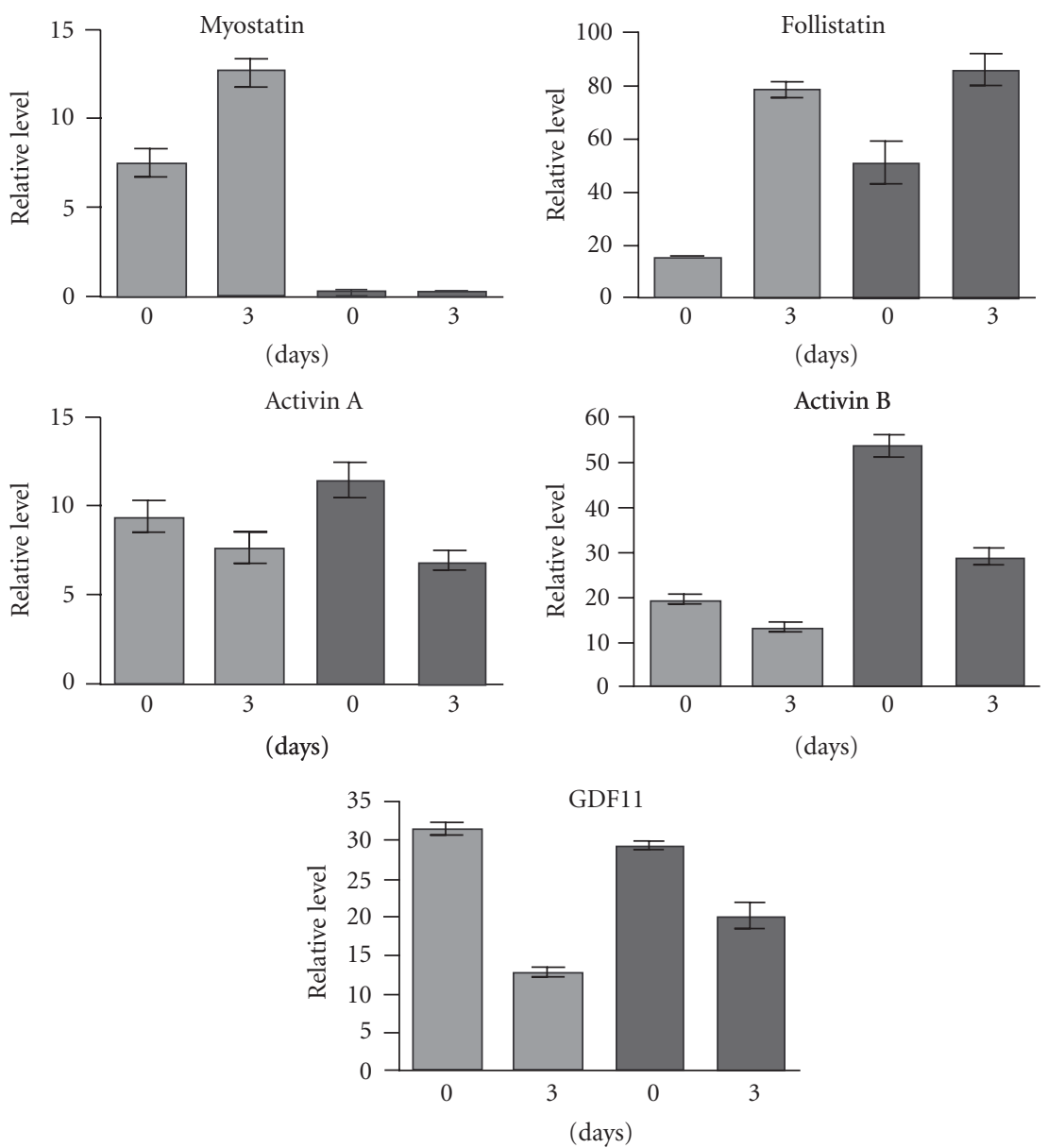

$\mathrm{C} 2 \mathrm{C} 12$

L6E9

(b)

FIGURE 4: (a) Semiquantitative RT-PCR analysis was carried out to detect the transcript levels of myostatin, follistatin, Activins A and B, and GDF11 in C2C12 and L6E9 myoblasts, undifferentiated (day 0) or differentiated (day 3). (b) The graphs compare the relative expression levels of each transcript between C2C12 and L6E9 myoblasts, undifferentiated (day 0) and differentiated (day 3), as obtained after normalization with gapdh $(n=3)$. 
Morphologically, each treatment was effective to promote a remarkable increase of L6E9 differentiation, characterized by formation of myotubes that were significantly larger compared to control (Figure 3(b)). As shown by graphical representation (Figure 3(c)), delivery of either dnActRIIb or follistatin produced a significant increase of the average size compared to control; after TSA treatment, the differences in myotube size became statistically significant after day 2 of treatment compared to control. Under these conditions, the protein levels of myogenin, Cav-3, and MyHC were remarkably increased, although with different timing, compared to untreated cells (Figure 3(d)).

Collectively, these data confirm that the regulation of size in L6E9 myotubes occurs regardless of myostatin but still under the control of additional TGF- $\beta$ members, because either blunting the ActRIIs signaling or increasing follistatin expression enhanced the differentiation and further improved the development of myotubes in vitro, as occurs in vivo $[15,19]$.

3.3. L6E9 Myoblasts Express the TGF- $\beta$ Family Members Activins and GDF11. Myostatin signals into myoblasts through a canonical TGF- $\beta$ pathway, by eliciting the formation of a ternary Smad complex that can drive to gene transcription in collaboration with coactivators and repressors [25]. Therefore, the regulators that potentially display a myostatin-like activity presumably activate this pathway, whereas myostatin antagonists, such as follistatin, can antagonize it. The superfamily of TGF- $\beta$ includes over 50 structurally related ligands that belong to three major subfamilies, which are TGF- $\beta$, bone morphogenetic proteins (BMPs), and Activins [26]. Recently, Activins A and B have received a growing attention for their ability to regulate the fiber size [20,27]. Activins and myostatin share the binding site on ActRIIs, suggesting that they can eventually synergize to activate the Smad pathway [28]. Additionally, another member of TGF- $\beta$ family, termed GDF11 (Growth/Differentiation Factor 11), shares 90\% aminoacid identity with myostatin in carboxy-terminal region [20]. Therefore, the expression of Activins and GDF11 was evaluated by means of semiquantitative RT-PCR analysis in both L6E9 and $\mathrm{C} 2 \mathrm{C} 12$ myoblasts. As shown by gel analysis (Figure 4(a)) and by relative band quantification (Figure 4(b)), myostatin was found to increase exclusively in $\mathrm{C} 2 \mathrm{C} 12$ cells, whereas follistatin increased over the differentiation of both the cell lines. Under these conditions, the expression of Activins A, B, and GDF11 was detectable in both $\mathrm{C} 2 \mathrm{C} 12$ and L6E9 myoblasts; in particular, during the differentiation, GDF11 and Activin B levels were decreased in both the cell lines, whereas Activin A was unaffected. Taken together, these data suggest that L6E9 cells, despite lack myostatin, express Activins and GDF11, which could be potentially involved in the regulation of myotube size.

\section{Conclusions}

In this work, we provide evidence that L6E9 myoblasts are spontaneously lacking myostatin. Additionally, we have verified that the size of L6E9 myotubes can be incremented through delivery of a dominant-negative ActRIIb form or increase of follistatin, confirming that multiple TGF- $\beta$ regulators converge on ActRIIs receptors to determine the size of myofibers [19-21, 28, 29] and, importantly, that follistatin can neutralize most of them $[19,20,22,29,30]$. In addition to myostatin, GDF11, Activins (A and B) [29, 31], and BMP-9 and 10 proteins have been identified to bind ActRIIs [31]. However, whereas BMP-9 and 10 seem to convert C2C12 myoblasts in the osteoblast lineage [20], GDF11 and Activins exert an inhibitory effect on myocyte differentiation similar to myostatin, and their activity is neutralized by follistatin $[22,27]$. Recently, generation of mice with ablation of both myostatin and GDF11 provided evidence that these two highly related TGF- $\beta$ members have redundant and overlapping roles in regulating skeletal patterning but not in regulating muscle size [32]. Therefore, in addition to myostatin, Activins seem to play a major role in regulating the myofiber size. In line with these observations, it would be of interest to use L6E9 myoblasts for dissecting the contribute of myostatin-like molecules, such as Activins, GDF11, or specific BMP proteins, or follistatin-like molecules, such as decorin [33], which have the ability to neutralize multiple negative regulators. Overall, we propose L6E9 myoblasts as a suitable myostatin knock-out in vitro model to study the mechanisms underlying the developmental regulation of muscle fibers.

\section{Abbreviations}

DMEM: Dulbecco's modified eagle's medium

FBS: $\quad$ Fetal bovine serum

HS: $\quad$ Horse serum

PBS: $\quad$ Phosphate buffered solution

TGF- $\beta$ : Transforming growth factors beta.

\section{Acknowledgments}

The authors thank SJ. Lee and A. McPherron for kindly providing the MDAF2 vector harboring the dominant-negative ActRIIb form and Vittorio Sartorelli for the $\mathrm{pBabe/follistatin}$ vector. This work was supported by grants from $60 \%$ MIUR to A.F., PRIN 2007 (2007XT947K contract), and by grants from "Amici per il cuore".

\section{References}

[1] A. C. McPherron, A. M. Lawler, and S.-J. Lee, "Regulation of skeletal muscle mass in mice by a new TGF- $\beta$ superfamily member," Nature, vol. 387, no. 6628, pp. 83-90, 1997.

[2] S.-J. Lee and A. C. McPherron, "Myostatin and the control of skeletal muscle mass," Current Opinion in Genetics and Development, vol. 9, no. 5, pp. 604-607, 1999.

[3] S.-J. Lee, "Regulation of muscle mass by myostatin," Annual Review of Cell and Developmental Biology, vol. 20, pp. 61-86, 2004.

[4] K. Patel and H. Amthor, "The function of Myostatin and strategies of Myostatin blockade-new hope for therapies aimed at promoting growth of skeletal muscle," Neuromuscular Disorders, vol. 15, no. 2, pp. 117-126, 2005. 
[5] D. Joulia-Ekaza and G. Cabello, "Myostatin regulation of muscle development: molecular basis, natural mutations, physiopathological aspects," Experimental Cell Research, vol. 312, no. 13, pp. 2401-2414, 2006.

[6] A. C. McPherron and S.-J. Lee, "Double muscling in cattle due to mutations in the myostatin gene," Proceedings of the National Academy of Sciences of the United States of America, vol. 94, no. 23, pp. 12457-12461, 1997.

[7] D. S. Mosher, P. Quignon, C. D. Bustamante, et al., "A mutation in the myostatin gene increases muscle mass and enhances racing performance in heterozygote dogs," PLoS Genetics, vol. 3, no. 5, p. e79, 2007.

[8] M. Schuelke, K. R. Wagner, L. E. Stolz, et al., "Myostatin mutation associated with gross muscle hypertrophy in a child," The New England Journal of Medicine, vol. 350, no. 26, pp. 2682-2688, 2004.

[9] T. A. Zimmers, M. V. Davies, L. G. Koniaris, et al., "Induction of cachexia in mice by systemically administered myostatin," Science, vol. 296, no. 5572, pp. 1486-1488, 2002.

[10] C. J. Carlson, F. W. Booth, and S. E. Gordon, "Skeletal muscle myostatin mRNA expression is fiber-type specific and increases during hindlimb unloading," American Journal of Physiology, vol. 277, no. 2, pp. R601-R606, 1999.

[11] N. F. Gonzalez-Cadavid, W. E. Taylor, K. Yarasheski, et al., "Organization of the human myostatin gene and expression in healthy men and HIV-infected men with muscle wasting," Proceedings of the National Academy of Sciences of the United States of America, vol. 95, no. 25, pp. 14938-14943, 1998.

[12] K. Ma, C. Mallidis, S. Bhasin, et al., "Glucocorticoid-induced skeletal muscle atrophy is associated with upregulation of myostatin gene expression," American Journal of Physiology, vol. 285, no. 2, pp. E363-E371, 2003.

[13] J. Massague, "How cells read TGF- $\beta$ signals," Nature Reviews Molecular Cell Biology, vol. 1, no. 3, pp. 169-178, 2000.

[14] J. L. Wrana, "Regulation of Smad activity," Cell, vol. 100, no. 2, pp. 189-192, 2000.

[15] S.-J. Lee and A. C. McPherron, "Regulation of myostatin activity and muscle growth," Proceedings of the National Academy of Sciences of the United States of America, vol. 98, no. 16, pp. 9306-9311, 2001.

[16] J. J. Hill, M. V. Davies, A. A. Pearson, et al., "The myostatin propeptide and the follistatin-related gene are inhibitory binding proteins of myostatin in normal serum," The Journal of Biological Chemistry, vol. 277, no. 43, pp. 40735-40741, 2002.

[17] H. Amthor, G. Nicholas, I. McKinnell, et al., "Follistatin complexes Myostatin and antagonises Myostatin-mediated inhibition of myogenesis," Developmental Biology, vol. 270, no. 1, pp. 19-30, 2004.

[18] S. Iezzi, M. Di Padova, C. Serra, et al., "Deacetylase inhibitors increase muscle cell size by promoting myoblast recruitment and fusion through induction of follistatin," Developmental Cell, vol. 6, no. 5, pp. 673-684, 2004.

[19] S.-J. Lee, "Quadrupling muscle mass in mice by targeting TGF$\beta$ signaling pathways," PLoS One, vol. 2, no. 8, article e789, 2007.

[20] T. A. Souza, X. Chen, Y. Guo, et al., "Proteomic identification and functional validation of activins and bone morphogenetic protein 11 as candidate novel muscle mass regulators," Molecular Endocrinology, vol. 22, no. 12, pp. 2689-2702, 2008.

[21] Y. Xia and A. L. Schneyer, "The biology of activin: recent advances in structure, regulation and function," Journal of Endocrinology, vol. 202, no. 1, pp. 1-12, 2009.
[22] A. L. Schneyer, Y. Sidis, A. Gulati, J. L. Sun, H. Keutmann, and P. A. Krasney, "Differential antagonism of activin, myostatin and growth and differentiation factor 11 by wild-type and mutant follistatin," Endocrinology, vol. 149, no. 9, pp. 45894595, 2008.

[23] D. L. Allen and T. G. Unterman, "Regulation of myostatin expression and myoblast differentiation by FoxO and SMAD transcription factors," American Journal of Physiology, vol. 292, no. 1, pp. C188-C199, 2007.

[24] M. Thomas, B. Langley, C. Berry, et al., "Myostatin, a negative regulator of muscle growth, functions by inhibiting myoblast proliferation," The Journal of Biological Chemistry, vol. 275, no. 51, pp. 40235-40243, 2000.

[25] Y. Shi and J. Massagué, "Mechanisms of TGF- $\beta$ signaling from cell membrane to the nucleus," Cell, vol. 113, pp. 685-700, 2003.

[26] X. H. Feng and R. Derynck, "Specificity and versatility in TGF- $\beta$ signaling through Smads," Annual Review of Cell \& Developmental Biology, vol. 21, pp. 659-693, 2005.

[27] H. Gilson, O. Schakman, S. Kalista, P. Lause, K. Tsuchida, and J.-P. Thissen, "Follistatin induces muscle hypertrophy through satellite cell proliferation and inhibition of both myostatin and activin," American Journal of Physiology, vol. 297, no. 1, pp. E157-E164, 2009.

[28] P. C. Gray, J. Greenwald, A. L. Blount, et al., "Identification of a binding site on the type II activin receptor for activin and inhibin,” The Journal of Biological Chemistry, vol. 275, no. 5, pp. 3206-3212, 2000.

[29] S.-J. Lee, L. A. Reed, M. V. Davies, et al., "Regulation of muscle growth by multiple ligands signaling through activin type II receptors," Proceedings of the National Academy of Sciences of the United States of America, vol. 102, no. 50, pp. 18117-18122, 2005.

[30] S. Abe, M. Soejima, O. Iwanuma, et al., "Expression of myostatin and follistatin in mdx mice, an animal model for muscular dystrophy," Zoological Science, vol. 26, no. 5, pp. 315320, 2009 .

[31] K. Tsuchida, M. Nakatani, A. Uezumi, T. Murakami, and X. Cui, "Signal transduction pathway through activin receptors as a therapeutic target of musculoskeletal diseases and cancer," Endocrine Journal, vol. 55, no. 1, pp. 11-21, 2008.

[32] A. C. McPherron, T. V. Huynh, and S.-J. Lee, "Redundancy of myostatin and growth/differentiation factor 11 function," BMC Developmental Biology, vol. 9, no. 1, article 24, 2009.

[33] J. Zhu, Y. Li, W. Shen, et al., "Relationships between transforming growth factor- $\beta 1$, myostatin, and decorin: implications for skeletal muscle fibrosis," The Journal of Biological Chemistry, vol. 282, no. 35, pp. 25852-25863, 2007. 

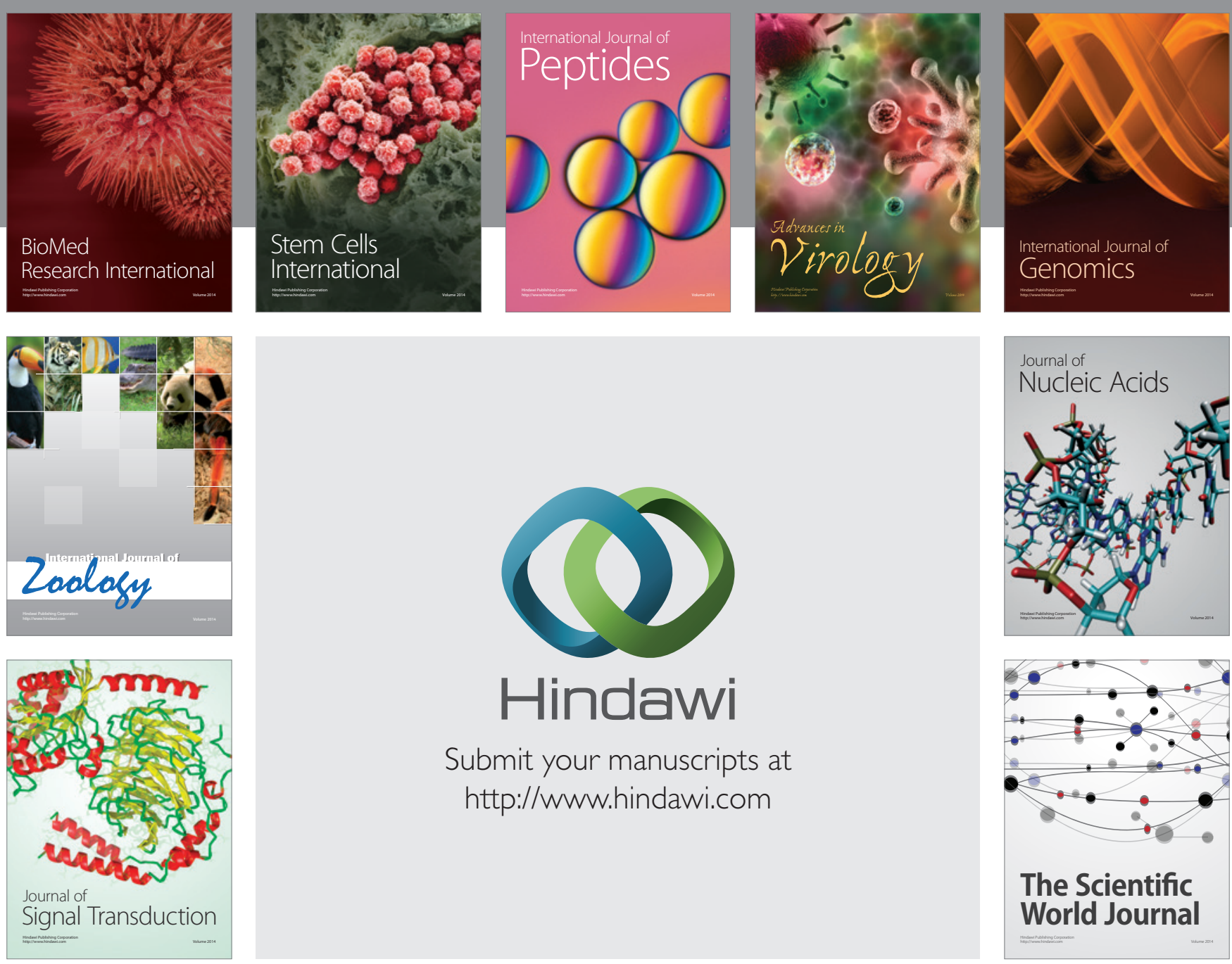

Submit your manuscripts at

http://www.hindawi.com
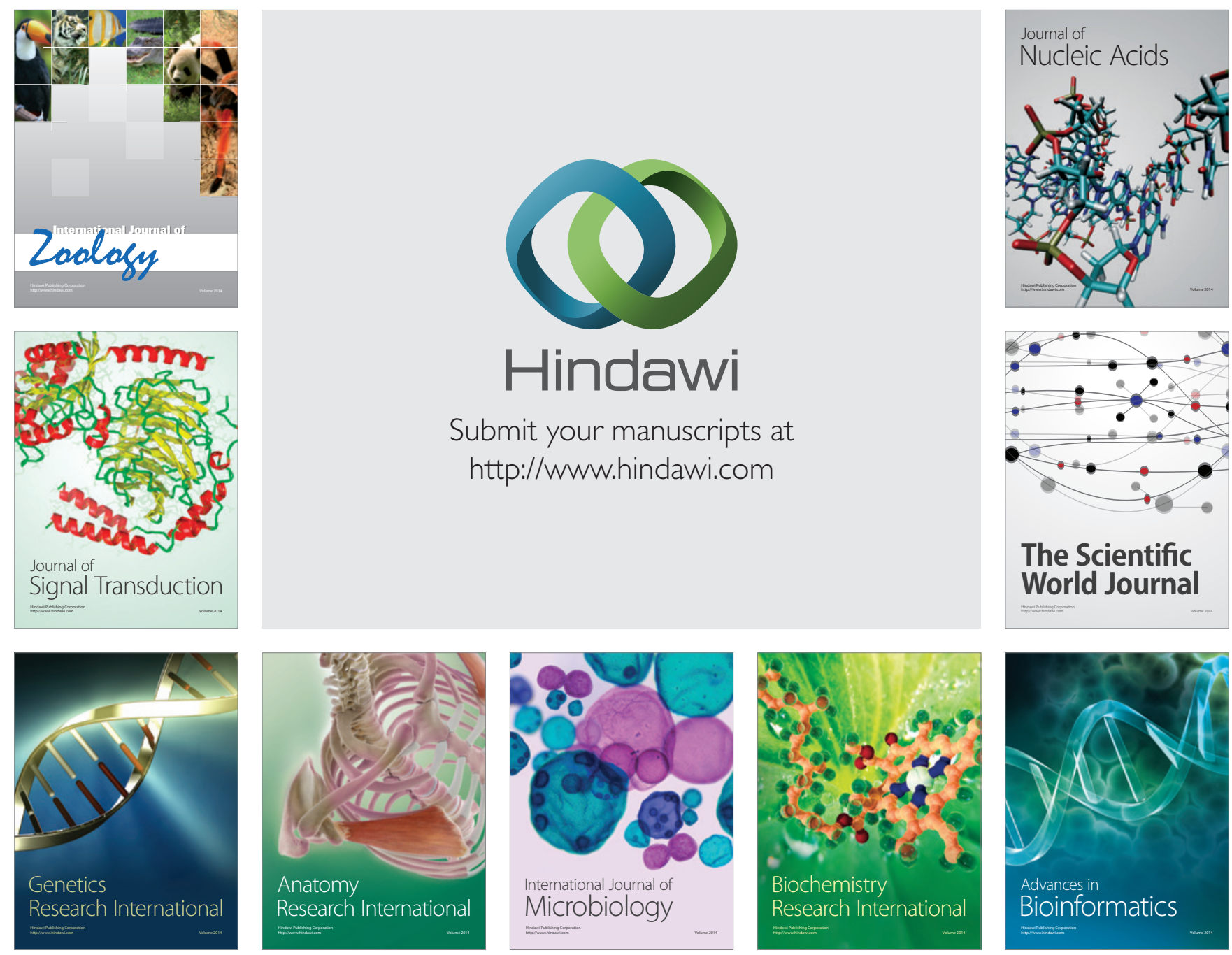

The Scientific World Journal
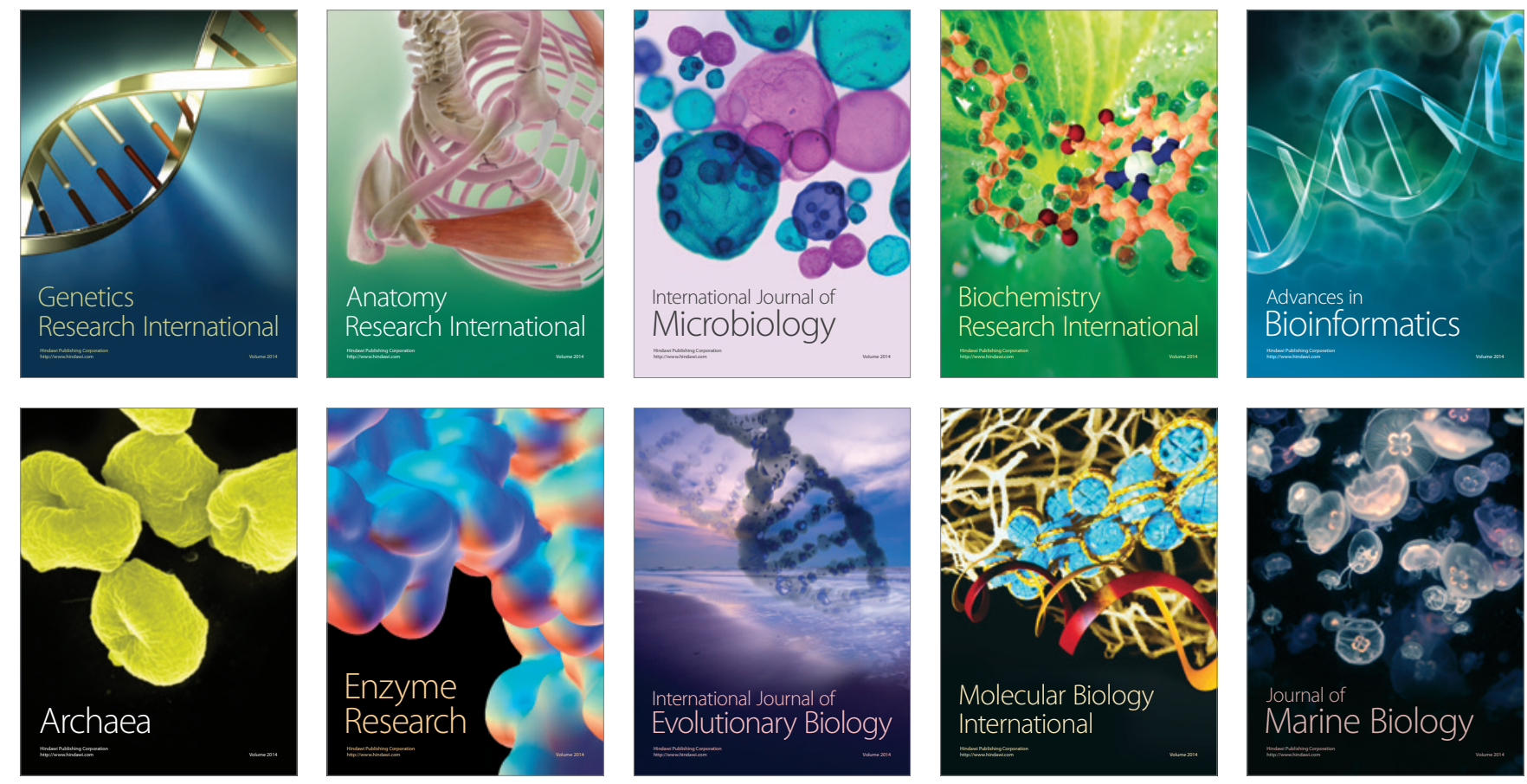